\title{
Rejet du genre Pseudostrigea Yamaguti, 1933 (Trematoda : Strigeidae)
}

\author{
Par Georges DUBOIS
}

Yamaguti (1933, pp. 3 et 6) créa le genre Pseudostrigea avec $P$. buteonis (1) comme type, de Buteo buteo japonicus Temm. et Schleg. [syn. de Buteo burmanicus Hume, d'après Peters]. En 1939, il retrouvait l'espèce dans Circus aeruginosus (L.) et dans Spizaëtus nipalensis orientalis Temm. et Schleg.

D'après la description originale et la figure 1 qui l'illustre, et en dépit de l'hébergement par des Falconiformes, il s'agit d'un Apatemon typique, caractérisé par des testicules en forme de rein, de cœur ou de fraise (probablement trilobés antérieurement) et orientés plus ou moins obliquement (surtout le premier). Le même galbe a été observé pour les testicules d'A patemon gracilis gracilis (Rud.) (Yamaguti 1933, fig. 2), d'Apatemon gracilis somateriae Dub. (Dubois 1948, fig. 1; Dubois et Rausch 1960, fig. 1), d'Apatemon gracilis canadensis Dub. et Rsch. (Dubois et Rausch 1948, p. 35, fig. $2 ; 1950$, p. 33) et d'Apatemon gracilis congolensis Dub. et Fain (Dubois et Fain 1956, fig. 11). D'ailleurs, chez les Apatemon d'Anatidés, les testicules ont toujours été décrits comme des organes arrondis, ovales ou cordiformes, non ou peu profondément lobés [sauf chez A. intermedius (S. J. Johnston, 1904), où la lobation est plus profonde, le contour restant arrondi (cf. T. H. Johnston et L. M. Angel 1951, fig. 1 et 2)].

Pour justifier l'érection de son nouveau genre, Yamaguti considérait le cône génital bien différencié, « large and well demarcated» $(1933$, p. 6). Or, cette particularité ne ressort pas de la figure 1 , mais elle se retrouve chez $A$. intermedius (cf. Johnston et Angel, op. cit., fig. 4 et 14), chez A. gracilis canadensis (cf. Dubois et Rausch 1948, fig. 2) et chez A. gracilis congolensis (cf. Dubois et Fain, op. cit., fig. 11).

Il n'est donc plus possible de maintenir le genre Pseudostrigea, ni de le ramener au rang de sous-genre d'Apatemon, comme nous l'avons fait (1953, pp. 40, 42), puisque le type est un vrai Apatemon (à court canal hermaphrodite) (2).

Parmi les espèces prétendues congénériques (ou subcongénériques) se trouvait Pseudostrigea sarcogyponis Vidyarthi, 1937, de Sarcogyps calvus (Scop.), caractérisé

(1) Mal orthographié (butenois) à la page 3 .

(2) Nous n'avons reçu aucune réponse à la demande de prêt de l'holotype ou du paratype de Pseudostrigea buteonis. 
par des testicules multilobés, développés transversalement et confinés dans la seconde moitié du segment postérieur. L'ovaire est situé à mi-longueur de ce dernier, et les vitellogènes se terminent devant le cône génital (à $0,8 \mathrm{~mm}$ de l'extrémité du corps). La tôt les apparences d'une poche éjaculatrice, mais qui occupe l'emplacement d'un cône génital. Le Ver mesure 4,38 à $6,48 \mathrm{~mm}$.

La multilobation des testicules peut constituer un caractère distinctif permettant figure $\mathrm{F}$ de la description originale montre un « ductus ejaculatorius » (D.ej.) qui a plud'opposer l'espèce sarcogyponis aux Apatemon s. str., qui ont des gonades mâles en forme de rein, de cœur ou de fraise, avec découpure antérieure en trois lobes plus ou moins distincts et une légère orientation dorsale de leur axe longitudinal. La question reste ouverte.

On a pu rapprocher de sarcogyponis le Strigéide indien que Odening (1962, pp. 383 386 , fig. $1 a-b$ ) a décrit sous le nom d'Apatemon (Pseudostrigea?) parapandubi. Il provient de Bubulcus ibis coromandus (Bodd.) et de Pelecanus philippensis Gm. Sur l'holotype que nous avons eu à l'examen (préparation kT 1/89, de Pelecanus), les testicules sont multilobés (fig. 1) et situés « etwa in der Mitte des Hinterkörpers oder etwas weiter hinten ». Sur l'exemplaire dessiné par Zieger in Odening (kT 1/95, de Bubulcus, fig. $1 b$ ), que nous reproduisons ici (fig. 2), on discerne des vitellogènes dans le segment antérieur, en diffusion discrète jusqu'au niveau de la ventouse ventrale, alors que la densité folliculaire est forte dans le segment postérieur. (Cette répartition rappelle celle de Apharyngostrigea indiana Vidyarthi, 1937). La glande protéolytique est très développée, comme chez Apharyngostrigea ardeolina Vidyarti, 1937 ou chez A. ramai (Verma, 1936), avec la même structure (masse basale postérieure et lobules antérieurs). Le pharynx paraît absent, et on distingue deux petits lobes dorsaux de l'organe tribocytique, insérés derrière la ventouse ventrale, tels qu'ils ont été observés et dessinés plusieurs fois par Odening chez des espèces du genre Apharyngostrigea. Les ventouses sont relativement petites. Le Ver mesure 2,2 à $4 \mathrm{~mm}$.

Une autre espèce, Apatemon pandubi Pande, 1939 avait été attribuée au sousgenre Pseudostrigea (cf. Dubois 1938, pp. 40 et 42). Elle est caractérisée par le développement asymétrique du premier testicule et par la forme bilobée et en fer à cheval du second. L'hôte est un Pélécaniforme (Anhinga melanogaster Penn.) [Inde]. Ce parasite a été retrouvé en Australie chez Phalacrocorax sulcirostris (Brandt) (Dubois et Pearson, 1965) (fig. 3). Il a été transféré dans le genre Schwartzitrema Vigueras, avec Apatemon truonis Dubois et Fain, 1956 (3) et Schwartzitrema nigericus R. Gupta, 1962 comme synonymes.

Schwartzitrema pandubi (Pande) possède un cône génital traversé par le canal hermaphrodite, comme chez les Apatemon (cf. Dubois et Fain 1956, fig: 10 ; R. Gupta, 1962, fig. 1 et 3). Mais la morphologie des testicules (comparables à ceux des Diplostomes) et l'inféodation aux Pélécaniformes justifient ce transfert.

(3) Nous avions considéré $A$. truonis comme distinct de $A$. pandubi car les vitellogènes du premier s'étendent jusqu'à l'équateur du cône génital, tandis que ceux du second, selon Pande, ne dépasseraient pas le milieu du testicule postérieur. On ne peut plus faire cette distinction, puisque Gupta observe ces glandes jusque dans la région de la bourse copulatrice de nigericus. 


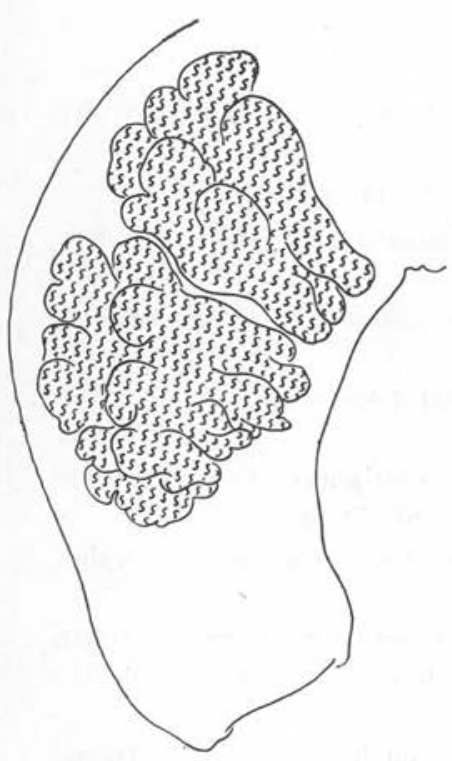

FIG. 1

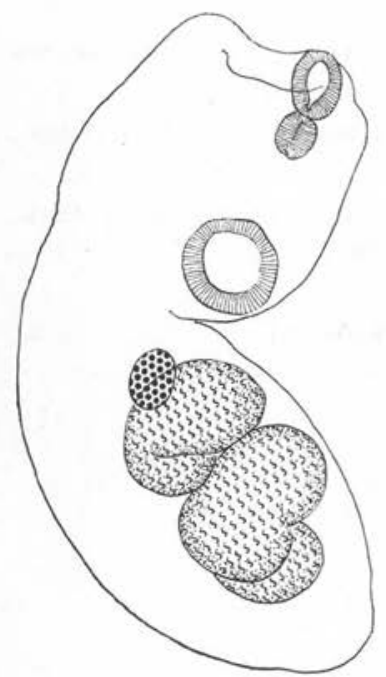

FIG. 3

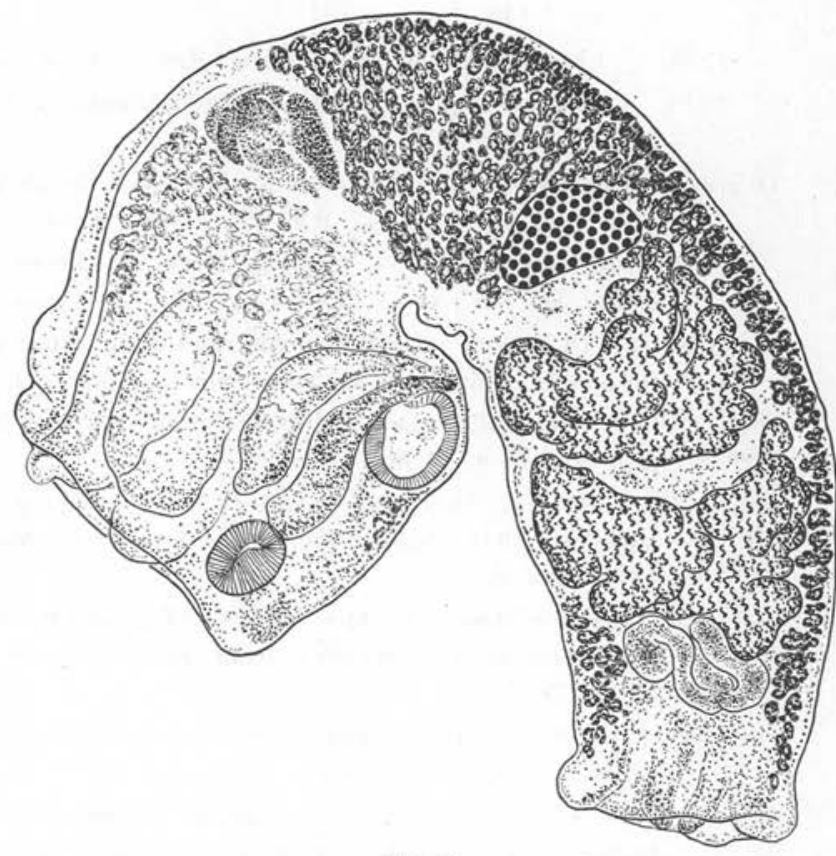

FIG. 2

Fig. 1. - «Apatemon (Pseudostrigea?) parapandubi» Odening, 1962, de Pelecanus philippensis Gm. Holotype (Coll. Odening, kT 1/89). Morphologie des testicules.

FIG. 2. - «Apatemon (Pseudostrigea?) parapandubi » Odening, 1962, de Bubulcus ibis coromandus (Bodd.). (Coll. Odening, kT 1/95). Longueur 2,5 mm. (La densité des vitellogènes est faible dans le segment antérieur.) Fig. 3. - Schwartzitrema pandubi (Pande, 1939), de Phalacrocorax sulcirostris (Brandt). (Coll. Pearson, $\mathrm{N}^{\circ}$ 440). Longueur $1,45 \mathrm{~mm}$. Morphologie des testicules. 


\section{Bibliographie}

Dubors (G.), 1938. - Monographie des Strigeida (Trematoda). Mém. Soc. neuchâtel. Sci. nat. $6: 1-535,354$ fig.

—, 1948. — Liste des Strigéidés de Suisse. Rev. suisse Zool. 55 : 447-476, 4 fig.

-, 1953. - Systématique des Strigeida. Complément de la Monographie. Mém. Soc. neuchâtel Sci. nat. 8 (2): 1-141.

Dubors (G.) et Fain (A.), 1956. - Contribution à l'étude des Strigeida du Congo belge. I. Bull. Soc. neuchâtel Sci. nat. 79: 17-38, 16 fig.

-, et Pearson (J.-C), 1965. - Quelques Strigeida (Trematoda) d'Australie. Bull. Soc. neuchâtel. Sci. nat., 88 : 77-99, 14 fig.

—, et Rausch (R.), 1948. - Seconde contribution à l'étude des « Strigeides 》 ( Trematoda ») nord-américains. Bull. Soc. neuchâtel. Sci. nat., 71 : 29-61, 21 fig.

—, —, 1960. — Quatrième contribution à l'étude des Strigeides (Trematoda) nord-américains. Ibid. 83 : 79-92, 3 fig.

Gupta (R.), 1962. - Two new species of the rare genus Schwartzitrema (Vigueras, 1940) Vigueras, 1941 (Trematoda: Strigeidae). Proc. Nat. Acad. Sci. India, sect. B, 32 : 387-392, 4 fig.

Johnston (T. H.) et ANGel (L. M.), 1951. - The morphology and life cycle of the Trematode, Apatemon intermedius, from the black swan. Trans. Roy. Soc. S. Austr., 74 : 66-78, 28 fig.

Odening (K.), 1962. - Trematoden aus Indischen Vögeln des Berliner Tierparks. Z. f. Parasitenk. $21: 381-425,26$ fig.

PANDE (B. P.), 1939. - Two new species of Trematodes from Anhinga melanogaster, the Indian darter or snake-bird. Proc. Nat. Acad. Sci. India 9: 22-28, 4 fig.

VIDYARTHI (R. D.), 1937. - New avian Trematodes of the sub-subfamily Cotylurini Dubois, 1936 (Family Strigeidae Railliet, 1919). Proc. Ind. Acad. Sci. $5: 315-323,4$ fig.

Yamaguti (S.), 1933. - Studies on the helminth fauna of Japan. Part I. Trematodes of Birds, Reptiles and Mammals. Japan. J. Zool. 5: 1-134. 57 fig.

[M. G. Dubois, 2035 - Corcelles (Neuchâtel), Suisse] 